\title{
ARTICLE OPEN \\ Unfolding the complexity of phonon quasi-particle physics in disordered materials
}

Sai Mu (iD ${ }^{1,5,6 *}$, Raina J. Olsen ${ }^{1,6}$, Biswanath Dutta ${ }^{2}{ }^{2,6}$, Lucas Lindsay ${ }^{1}$, German D. Samolyuk ${ }^{1}$, Tom Berlijn ${ }^{3,4}$, Eliot D. Specht ${ }^{1}$, Ke Jin ${ }^{1}$, Hongbin Bei ${ }^{1}$, Tilmann Hickel $\mathbb{D}^{2}$, Bennet C. Larson ${ }^{1}$ and George M. Stocks ${ }^{1 *}$

The concept of quasi-particles forms the theoretical basis of our microscopic understanding of emergent phenomena associated with quantum-mechanical many-body interactions. However, the quasi-particle theory in disordered materials has proven difficult, resulting in the predominance of mean-field solutions. Here, we report first-principles phonon calculations and inelastic X-ray and neutron-scattering measurements on equiatomic alloys ( $\mathrm{NiCo}, \mathrm{NiFe}, \mathrm{AgPd}$, and $\mathrm{NiFeCo}$ ) with force-constant dominant disorderconfronting a key 50-year-old assumption in the Hamiltonian of all mean-field quasi-particle solutions for off-diagonal disorder. Our results have revealed the presence of a large, and heretofore unrecognized, impact of local chemical environments on the distribution of the species-pair-resolved force-constant disorder that can dominate phonon scattering. This discovery not only identifies a critical analysis issue that has broad implications for other elementary excitations, such as magnons and skyrmions in magnetic alloys, but also provides an important tool for the design of materials with ultralow thermal conductivities.

npj Computational Materials (2020)6:4; https://doi.org/10.1038/s41524-020-0271-3

\section{INTRODUCTION}

Quasi-particle elementary excitations, including electron quasiparticles, phonons, magnons, plasmons, excitons, etc., along with skyrmions, ${ }^{1,2}$ Majorana fermions, ${ }^{3}$ and their mutual interactions, represent remarkably successful theoretical descriptions of emergent phenomena associated with quantum-mechanical many-body interactions. For example, the seminal discovery of the quantized thermal Hall effect in the spin liquid candidate $\mathrm{RuCl}_{3}^{4,5}$ can be explained by the coupling of quasi-particles (phonons and chiral Majorana edge modes) in Kitaev's nonAbelian spin liquid. ${ }^{6,7}$ As such, quasi-particle physics has provided a microscopic understanding of the underlying science of phenomena ranging from the novel properties of quantum materials to the electronic and vibrational-based physics in solids. In contrast to fully ordered crystals that can be described within the Bloch theorem, the broken translational symmetry in alloys associated with substitutional chemical disorder has long challenged the development of analogous robust quasi-particle theories of configurationally averaged observables that inherently lead to the finite quasi-particle lifetimes measured experimentally - even at zero temperature. Therefore, describing both the spectral dispersion and lifetimes of quasi-particles in disordered materials is fundamentally important for understanding the underlying science of condensed matter and the design of technological materials with targeted properties.

Historically, a landmark advance in treating compositional disorder was the coherent potential approximation (CPA). ${ }^{8-10}$ As an analytic "single-site" theory of the configurationally averaged observables that restores the translational invariance of a selfconsistently determined effective medium, the CPA formalism was initially designed to treat on-site (site-diagonal) fluctuations associated with the different alloying elements-different on-site orbital energies for the case of electrons, different atomic masses for the case of phonons. Effective as the original CPA was, it was quickly realized that extending the single-site CPA to include fluctuations in two-site quantities-inter-site hopping integrals for electrons, pairwise force constants for phonons-is nontrivial. ${ }^{11-14}$ This problem is particularly acute for phonons where forceconstant (off-diagonal) disorder is crucial and entangled with the mass (diagonal) disorder, as illustrated in Fig. 1a. For electron quasi-particles, it was possible to account for both diagonal and off-diagonal fluctuations by reformulating CPA within the language of multiple scattering theory ${ }^{15}$ and ab initio density functional theory. ${ }^{16-18}$ For phonon quasi-particles, no similar "simple" transformation of the underlying theory has been possible.

For phonons, the formulation of itinerant CPA (ICPA) by Ghosh et al. ${ }^{19}$ provided a substantial advance in analytic meanfield theories of phonon quasi-particle physics in disordered materials based on the augmented space formalism of Mookerjee. ${ }^{20}$ The theory was demonstrated initially for the cases of NiPd and NiPt that contain large mass disorder as well as force-constant disorder, ${ }^{19,21}$ and has been applied subsequently to multiple binary alloys with similarly dominant mass disorder. ${ }^{22-25}$ Remarkably, with the exception of a limited study of face-centered cubic (fcc) $\mathrm{Ni}_{88} \mathrm{Cr}_{12}$ and bcc $\mathrm{Fe}_{53} \mathrm{Cr}_{47}$ using an augmented space recursion (ASR) approach, ${ }^{26,27}$ neither systematic theoretical calculations of phonon dispersions and linewidths nor the corresponding experimental measurements have been performed for materials exhibiting strong force-constant disorder with small mass disorder. This unsatisfactory situation regarding experimental validation of the theory is further complicated by the form of the underlying Hamiltonian upon which it is based. The Hamiltonian for the ICPA and all previous mean-field theories (including the CPA) for off-diagonal as well as diagonal disorder make use of the simplifying assumption that force-constant

\footnotetext{
${ }^{1}$ Materials Science and Technology Division, Oak Ridge National Laboratory, Oak Ridge, TN 37831, USA. ${ }^{2}$ Max-Planck-Institut für Eisenforschung GmbH, D-40237 Düsseldorf, Germany. ${ }^{3}$ Center of Nanophase and Material Science, Oak Ridge National Laboratory, Oak Ridge, TN 37831, USA. ${ }^{4}$ Computational Science and Engineering Division, Oak Ridge National Laboratory, Oak Ridge, TN 37831, USA; ${ }^{5}$ Present address: Materials Department, University of California, Santa Barbara, CA 93106, USA. ${ }^{6}$ These authors contributed equally: Sai Mu, Raina J. Olsen, Biswanath Dutta. *email: sai.mu1986321@gmail.com; stocksgm@ornl.gov
} 


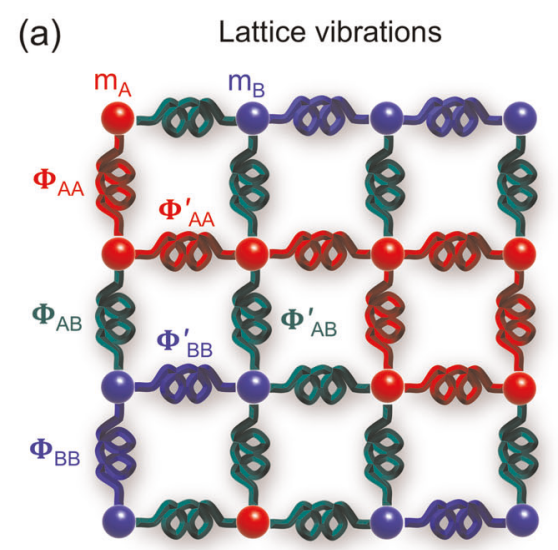

(c)

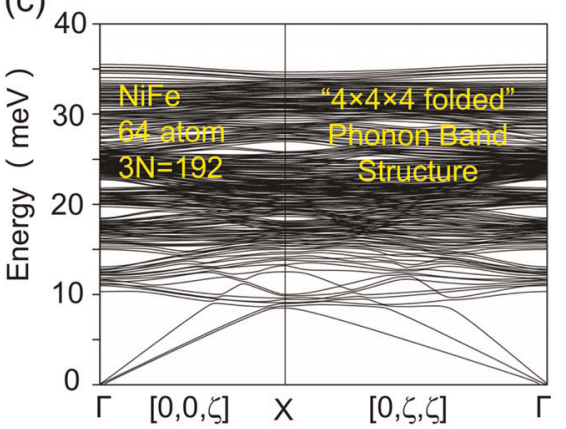

(b)

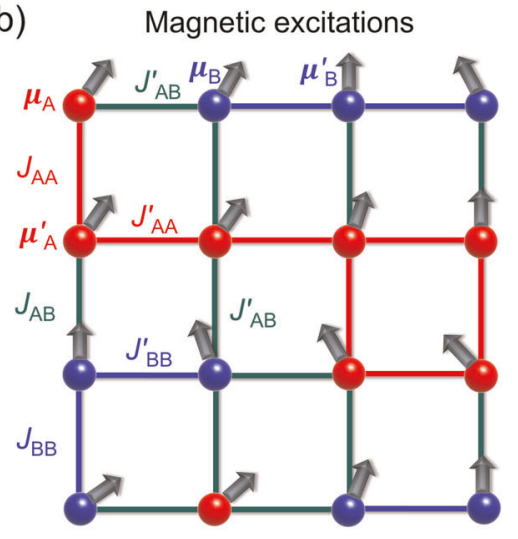

(d)

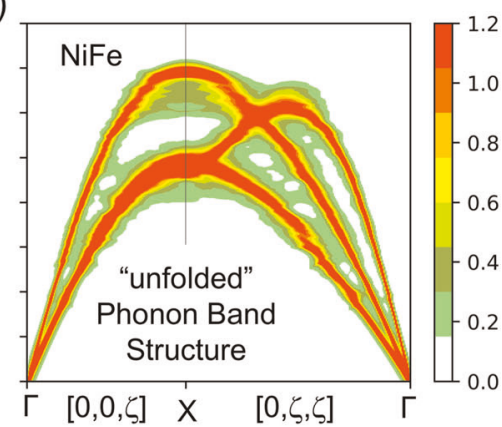

Fig. 1 Sketch of site-diagonal and off-diagonal disorder and phonon-unfolding method. Site-diagonal and off-diagonal disorder for a lattice vibrations and $\mathbf{b}$ magnetic excitations in random solid-solution alloys: $\mathrm{A}_{0.5} \mathrm{~B}_{0.5}$. The one-site quantities are the mass $\left(\mathrm{m}_{\mathrm{i}}\right)$ or local moment $\left(\mu_{i}\right)$ on site $i$, while the two-site quantities include the force constant $\left(\Phi_{i j}\right)$ or the exchange interaction $\left(J_{i j}\right)$ between sites $i$ and $j$. Primes indicate $\Phi^{\prime}{ }_{\mathrm{ij}} \neq \Phi_{\mathrm{ij}}$ due to different environments and the gray arrows in $\mathbf{b}$ denote the spin moments. (c) The folded phonon dispersion of a single 64 -atom $4 \times 4 \times 4$ supercell of NiFe along $[0,0, \zeta]$ and $[0, \zeta, \zeta]$ directions, where the wavevectors are collapsed by a factor of four compared with that for a primitive cell. $\mathbf{d}$ The unfolded phonon spectral function (arbitrary units) of a single 64 -atom $4 \times 4 \times 4$ supercell of NiFe along $[0,0, \zeta]$ and $[0, \zeta, \zeta]$ directions.

disorder between individual $A A, B B$, and $A B$-type pairs can be approximated by their global average in $A B$ binary alloys. ${ }^{13,28}$ Similar to lattice vibrations (phonons), the magnetic excitations (e.g., magnons) of disordered alloys are also challenging to describe due to site-diagonal (local moment) and off-diagonal (exchange interactions) disorder, as depicted in Fig. 1b. The crucial exchange interactions between individual $A A, B B$, and $A B$-type pairs are usually approximated by their ensemble averages as calculated from linear response theory. ${ }^{29}$ Unaccountably, although this 50 -year-old assumption within the Hamiltonian is at the core of the analytic mean-field formalisms, it has so far not been questioned.

In this work, we address this knowledge gap for the first time through a combined first-principles theory and experimental measurement investigation of the phonon quasi-particle physics (dispersions and linewidths) of concentrated disordered alloys, $\mathrm{NiCo}, \mathrm{NiFe}, \mathrm{AgPd}$, and $\mathrm{NiFeCo}$, with strong force-constant disorder but minimal mass disorder. Remarkably, we have discovered from $\mathrm{ab}$ initio supercell phonon-unfolding (SPU) simulations $\mathrm{s}^{30-32}$ and their comparison with ICPA and experimental measurements, that force-constant fluctuations considering each individual $A A, B B$, and $A B$-type species pairs far surpass that of the usual global average force-constant fluctuations. Moreover, we have shown that the source of the enhanced fluctuations is the inherent random variations in local chemical environments surrounding individual $A A, B B$, and $A B$-type pairs. Accordingly, we have demonstrated that the long-standing approximation of replacing individual-pair force-constant fluctuations with their global averages in the Hamiltonian of quasi-particle mean-field theories for disordered materials must be reconsidered.

\section{RESULTS AND DISCUSSION}

\section{Phonon properties of $\mathrm{NiCo}$ and $\mathrm{NiFe}$}

Phonon dispersion and linewidth measurements for NiCo and NiFe samples were performed at room temperature by using highresolution inelastic X-ray scattering (IXS) and inelastic neutronscattering (INS) measurements along the [001] and [011] reciprocal lattice directions. In addition, density functional theory (DFT) was employed to calculate the force constants of $\mathrm{NiCo}, \mathrm{NiFe}$, $\mathrm{AgPd}$, and NiFeCo alloys with chemical disorder modeled by the supercell method. Phonon spectral functions and the corresponding linewidths for equiatomic $\mathrm{NiCo}, \mathrm{NiFe}, \mathrm{AgPd}$, and $\mathrm{NiFeCo}$ alloys were extracted using both the ICPA ${ }^{19}$ and the SPU approach. ${ }^{32}$ We note that the analytical mean-field ICPA approach employs the symmetry-averaged force-constant matrix for a pair of atoms, i.e., one single matrix for each of $\Phi^{A A}, \Phi^{B B}$, and $\Phi^{A B}$ in binary $A-B$ alloys, while the nonanalytical SPU approach uses the full forceconstant matrix of the supercell. The SPU maps phonon bands within the Brillouin zone (BZ) of large supercells to those of the primitive BZ. While the phonon dispersion curves for an $\mathrm{N}$-atom supercell form $3 \mathrm{~N}$ continuous (sharp) phonon dispersion bands, when unfolded into the primitive BZ they map into three effective acoustic bands with finite-width distributions of discrete eigenvalues. This is illustrated in Fig. 1c, d. More experimental and calculation details can be found in the "Methods" section.

Figure 2 provides a color-contour overview of the phonon dispersions and phonon linewidths as obtained by SPU and ICPA calculations, along with the corresponding IXS and INS measurements for disordered solid-solution $\mathrm{NiCo}$ and $\mathrm{NiFe}$, respectively. These plots present results as a function of the wavevector $q=\left[\zeta_{x^{\prime}}\right.$ $\left.\zeta_{y}, \zeta_{z}\right]$ in reciprocal lattice units (rlu) of $2 \pi / a_{0}\left(a_{0}=\right.$ cubic lattice 

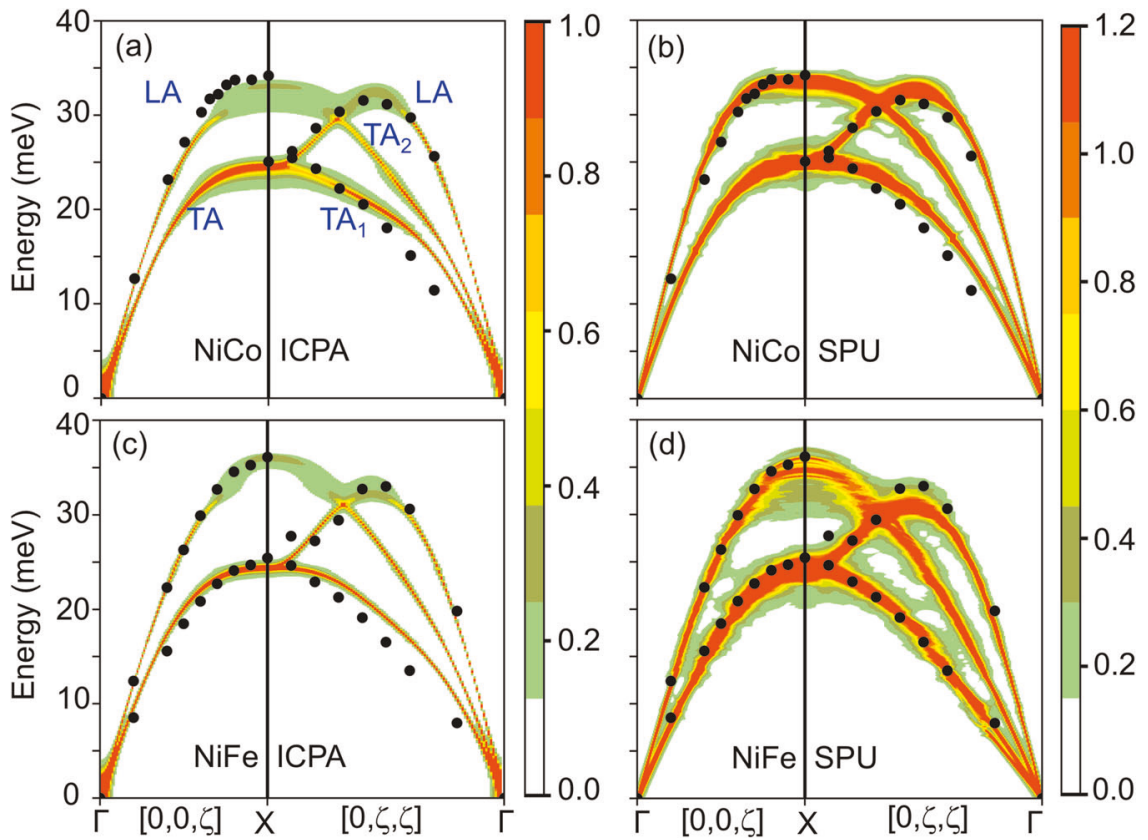

Fig. 2 Phonon dispersion along $[\mathbf{0}, \mathbf{0}, \boldsymbol{\zeta}]$ and $[\mathbf{0}, \boldsymbol{\zeta}, \boldsymbol{\zeta}]$ directions. Phonon spectral functions of $\mathbf{a}, \mathbf{b}$ NiCo and $\mathbf{c}, \mathbf{d}$ NiFe, obtained from ICPA and SPU separately. The measured data (solid circles) in the individual subfigures are compared with calculated phonon spectral functions (arbitrary units) from the SPU and ICPA calculations. The SPU phonon spectral functions are averaged over several different supercells.

parameter) for longitudinal (LA) and transverse (TA) acoustic phonons. The color contours in Fig. 2 give the simulated phonon spectral functions overlaid by discrete phonon dispersion measurements (black solid circles). We note general agreement between first-principles phonon dispersions (SPU and ICPA) and the corresponding measurements for both $\mathrm{NiCo}$ and NiFe, albeit small discrepancies occuring near the $[0,0, \zeta] B Z$ boundary $X$ point, and for $\mathrm{TA}_{1}$ phonons along the $[0, \zeta, \zeta]$ direction. Due to different normalizations used in the SPU and ICPA approaches, the magnitude of spectral functions is given in arbitrary units, and is therefore not directly comparable (as indicated in the different color bars). While this makes it difficult to compare visualizations, the different normalizations do not affect the peak position and disorder broadening of the spectral function, which are the focus of this work.

More important for disordered materials, though, are the phonon linewidths that provide a direct probe of force-constant disorder, when mass disorder is minimal. Figure 3 shows plots of the full width at half maximum (FWHM) linewidths, $\Delta$, of the SPU and ICPA spectral functions associated with the color contours in Fig. 2. These were obtained by fitting the spectral functions with Lorentzian profiles using negligible $(0.05 \mathrm{meV})$ resolution. Similarly, the linewidths corresponding to the measurements in Fig. 2 (open circles with error bars denoting uncertainties) were obtained by fitting the (constant q) IXS and INS spectral data with Lorentzian profiles convoluted with the $q$-resolution functions for the respective IXS and INS instruments. The IXS instrument resolution was determined directly by measurements of the elastic linewidth $(1.6 \mathrm{meV})$, and the $q$-dependent INS resolution was determined by RESLIB using the Popovici method. $^{33}$

We observe first of all, significant phonon broadening for both $\mathrm{NiCo}$ and NiFe in Fig. 3, especially for $q>0.7$ rlu. This broadening implies that force-constant disorder alone (in the absence of mass disorder) can cause significant phonon scattering, and hence, lead to shorter phonon lifetimes corresponding to substantial linewidth broadening. Furthermore, the measured linewidths are not only larger for NiFe than for NiCo, but dramatically so for $q<\sim 0.5$ rlu. The linewidths for NiCo tend to fall below $\sim 0.5 \mathrm{meV}$ for $q<0.5$ rlu, while the linewidths for NiFe range from 1.5 to $2 \mathrm{meV}$ down to $q$ as low as $\sim 0.2 \mathrm{rlu}$, the importance of which will be discussed below.

Focusing on the simulations, we observe in Fig. 3a that (within the measurement uncertainties) both the SPU and ICPA simulations agree with the measured linewidths for $\mathrm{NiCo}$, albeit ICPA tends to overestimate those of LA phonons in the $[0,0, \zeta]$ direction near the BZ boundary, and that the SPU simulations tend to underestimate the linewidths in the same region. In Fig. $3 b$, it is demonstrated that the linewidth simulations for NiFe using SPU are in excellent agreement with the measured linewidths for all wavevectors sampled. Conversely, the ICPA linewidths significantly underestimate the measured linewidths for NiFe-lower by more than a factor of two for the LA phonons and lower by factors of five to ten for the TA phonons.

Decomposition of disorder-induced phonon linewidths

To provide physical insight into the impact of force-constant disorder on phonon linewidths, we show by SPU simulations in Fig. $4 \mathrm{a}, \mathrm{b}$ the total and pair-resolved spectral functions for selected phonon modes for NiCo and NiFe. For small $q,[0,0,0.25]$ rlu, the individual-pair resolved and the total spectral functions for $\mathrm{NiCo}$ are quite sharp and symmetric, while for NiFe both the pairresolved and the total spectral functions are more than twice as broad. For larger $q,[0,0,0.75] \mathrm{rlu}$, the spectral functions for NiCo and NiFe are both broader and tend to be less symmetric, particularly for NiFe. For $q$ close to the X-point $[0,0,0.95]$ rlu, neither the NiCo nor the NiFe total spectral functions exhibit symmetric Lorentzian behavior. Instead, they comprise a sharp peak and a diffuse low-energy tail. The extended tails (together with the reduced intensity) on the low-energy sides for both $\mathrm{NiCo}$ and NiFe are analogous to those from ICPA investigations of alloys possessing both large mass and force-constant disorder. ${ }^{19,22-25}$ The tails result from the fact that the softer-like-species bondssuch as $\mathrm{Co}-\mathrm{Co}$ bonds in $\mathrm{NiCo}$ and $\mathrm{Ni}-\mathrm{Ni}$ bonds in NiFe (shown later)-do not vibrate resonantly at the high frequencies that occur for the other bonds at large wavevectors. Physically, the species-pair-dependent bond strengths can be understood within the context of the local electronic structure, the strength of 

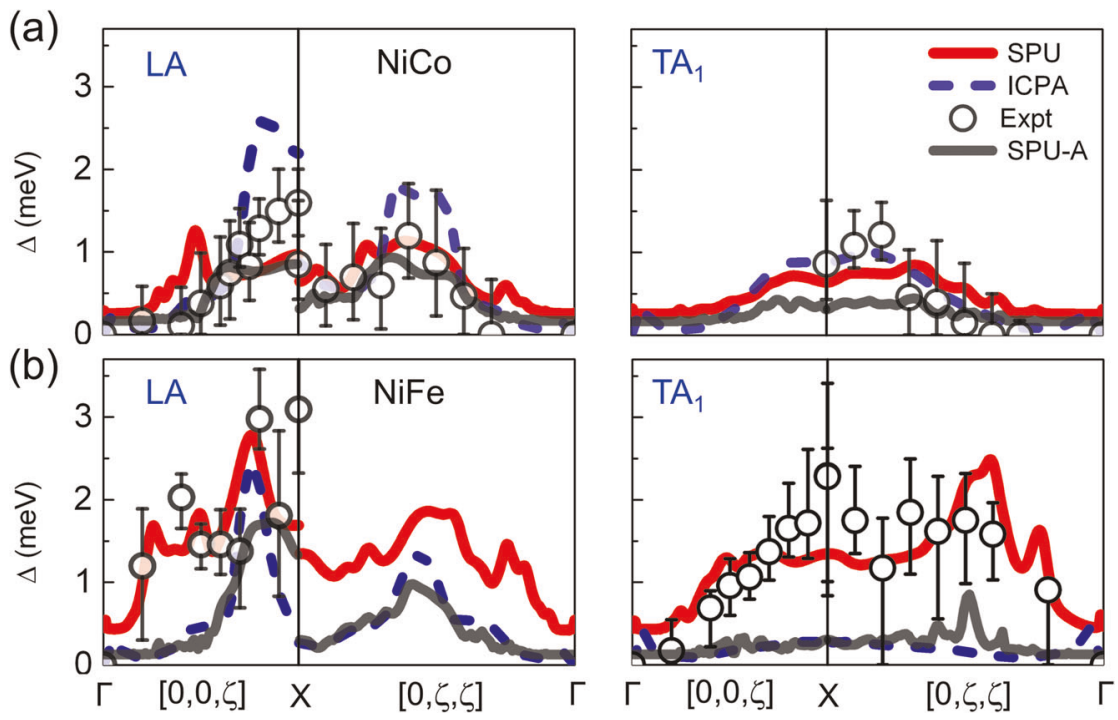

Fig. 3 Phonon linewidths of binary alloys versus wavevector. a NiCo and $\mathbf{b}$ NiFe. Phonon broadening is quantified by the FWHM linewidths $(\Delta)$ for $\mathrm{LA}$ and $\mathrm{TA}$, modes along $[0,0, \zeta]$ and $[0, \zeta, \zeta]$ directions. The measured linewidths are denoted by the black open circles, the solid red curves are linewidths from the SPU calculations, and the blue dashed curves correspond to the linewidths from ICPA. Linewidths from SPU-A are given by the translucent dark-gray curves. The measured linewidth uncertainties are proportional to the square root of the number of scattering events recorded at the given value of $\mathrm{E}, \mathrm{Q}$. Uncertainties in the inherent linewidths are determined by fitting a Lorentzian function convolved with the instrument resolution, then analyzing the chi-squared error of the fit as a function of the Lorentzian linewidth.

electron hybridization, and the occupations of the bonding and antibonding states, as discussed in Supplementary Note 1.3 and Supplementary Fig. 4.

Comparison between the SPU-calculated and the measured linewidths for $\mathrm{NiFe}$ and $\mathrm{NiCo}$ provides a direct validation of the SPU approach for predicting vibrational properties in disordered binary alloys where force-constant disorder dominates. This success now raises the question as to why the ICPA so significantly underestimates the linewidths in NiFe, given that the ICPA approach $^{19}$ was specifically designed to incorporate forceconstant disorder within an analytic mean-field theory.

Importance of off-diagonal randomness

To examine off-diagonal disorder, we have used SPU calculations to test the fundamental approximation within the present ICPA Hamiltonian for $A B$ binary alloys, ${ }^{19}$ which includes only the interspecies disorder between the "global averaged" force constants associated with individual species pairs $\left(<\Phi^{\mathrm{AA}}>\right.$, $\left\langle\Phi^{A B}\right\rangle,\left\langle\Phi^{B B}>\right.$ ). That is, ICPA averages over the actual distribution of force constants for individual species pairs, i.e., intraspecies force-constant disorder. While this approximation (in general use for mean-field quasi-particle theories) greatly simplifies the ICPA formalism and has underpinned attempts to generalize the original CPA to include off-diagonal randomness, ${ }^{13,28}$ it tacitly assumes that the local atomic environment surrounding interacting pairs does not impact the force constants for individual atomic pairs significantly. In contrast, however, we show in Fig. 5a, b that the impact of local chemical environments on the species-pair force constants is significant for $\mathrm{NiCo}$ and dramatically so for NiFe. Only the $\Phi_{x y}$ component of the force-constant matrix is shown in the figure, with the other components shown in Supplementary Note 1.1. Figure $5 \mathrm{a}, \mathrm{b}$ gives the individual (nearest neighbor) species-pair force constants for $\mathrm{NiCo}$ and $\mathrm{NiFe}$ as a function of the fractional distortion, $\delta r$, from the perfect crystal bond lengths. The individual force constants vary in two important aspects: (1) they tend to become softer for increasing bond lengths $\delta r$ according to the expected bond length proportionality, ${ }^{23,34,35}$ and (2) the force constants vary strongly in strength (in a statistical manner) for each bond length $\delta r$ due to a direct effect of random local chemical environment. We note that the variation of the bond lengths, as a result of atomic relaxation, is influenced by the local chemical environment. Therefore, the expected bond length proportionality represents an indirect effect of the local environment on the force constants. The histograms along the left vertical axes of Fig. 5a, b show the statistical distributions of the $\Phi^{\alpha \beta}$ for each species pair ( $a, \beta$ denote species), underscoring graphically the wider distributions of force constants for NiFe than for NiCo. Therefore, the local chemical environment clearly plays a critical role in determining species-pair force constants and their fluctuations, which in turn determine the linewidth behavior. In Fig. $5 \mathrm{a}, \mathrm{b}$ the average force constants $\left\langle\Phi^{\mathrm{a} \beta}\right\rangle$ (denoted by the yellow symbols) that represent the force-constant input to the ICPA Hamiltonian are also shown.

The thick gray SPU-A curves in Fig. 3 explicitly demonstrate the consequence of using averaged species-pair force constants. SPU$A$ replaces the individual $A A, A B$, and $B B$ species-pair force constants of the full SPU with their averages $\left(\left\langle\Phi^{A A}\right\rangle,\left\langle\Phi^{A B}\right\rangle\right.$, $\left\langle\Phi^{\mathrm{BB}}\right\rangle$ ), as detailed in Supplementary Note 1.2. Therefore, SPU-A is a supercell phonon-unfolding calculation that mimics the forceconstant averaging of the ICPA Hamiltonian. We note that the SPU-A, the full SPU, and the ICPA linewidth simulations are quite similar for NiCo. While the force-constant-averaged SPU-A calculations for NiFe depart strongly from those of SPU, they reproduce almost exactly the (anomalously low) ICPA linewidth results. These observations demonstrate conclusively that it is indeed the intraspecies force-constant averaging associated with the present ICPA Hamiltonian that is responsible for the breakdown in the ICPA linewidth predictions for NiFe. Conversely, the agreement between the SPU-A and the ICPA results indicates directly that the present ICPA properly accounts for the effects of off-diagonal disorder when it is limited to interspecies (averaged) randomness. It is important to note that the underestimated ICPA linewidths are due solely to the oversimplified Hamiltonian rather than the ICPA methodology itself.

This limitation was not apparent in previous ICPA alloy studies $^{19,22-25,36}$ because the impact of the large (100-150\%) mass disorder on phonon dispersion curves tended to mask the impact of force-constant disorder on phonon linewidths. In any case, the results presented above indicate that the incorporation 
(a)

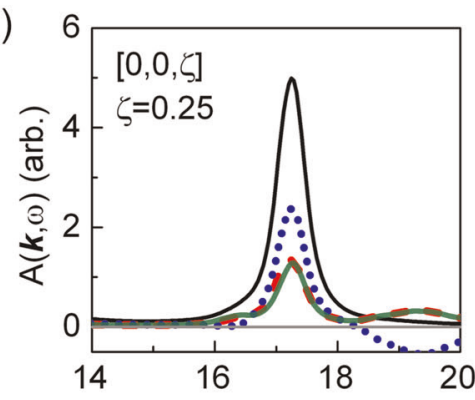

(b)

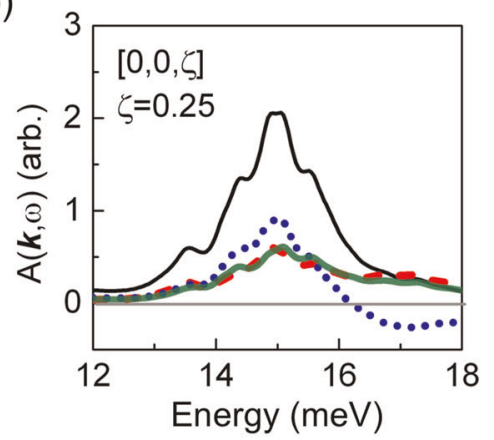

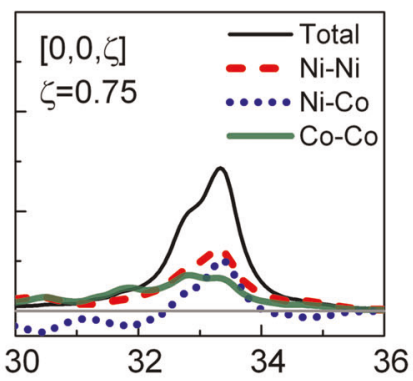
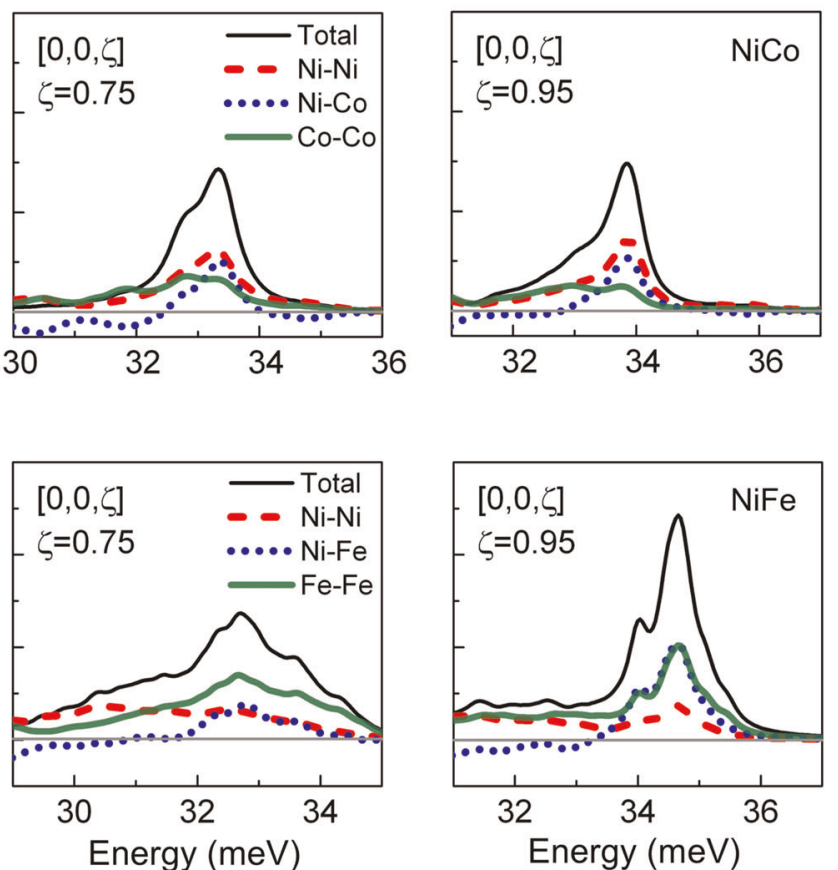

Fig. 4 Decomposition of phonon spectral functions. Total and species-pair-resolved partial spectral functions (arbitrary units) for LA modes in $\mathbf{a} \mathrm{NiCo}$ and $\mathbf{b} \mathrm{NiFe}$ at three $q$ points along the $[0,0, \zeta]$ direction. The gray horizontal line indicates zero.

of (individual) intraspecies force-constant fluctuations in the ICPA phonon Hamiltonian would lead to an accurate description of phonons in disordered materials. While outside the scope of this study, in principle the inclusion of individual-pair force-constant disorder in ICPA could be achieved by discretizing intraspecies force-constant distributions through the introduction of additional, appropriately weighted, ICPA components. This is similar to the discretization of the distribution of the relevant random variables (atomic displacements, spin disorder) by introducing more CPA components for configurational averaging in the electronic structure methodology. ${ }^{37}$

Phonon linewidths for other alloys with force-constant dominant disorder

To demonstrate that the importance of retaining full forceconstant distributions in $\mathrm{NiFe}$ is not an isolated case, we performed SPU and SPU-A phonon linewidth calculations for the equiatomic $4 d$ binary $\mathrm{AgPd}$ and the $3 d$ ternary NiFeCo alloys, both of which have negligible mass disorder (few percent). Figure $6 a$ compares the SPU-A, SPU, and ICPA linewidths for AgPd, demonstrating that the linewidth behaviors of SPU-A and ICPA are again similar, both of which differ strongly from the full SPU simulations for all phonon modes. Qualitatively different from $\mathrm{NiCo}$ and NiFe, the species-averaged force constants in AgPd are nearly equal (see Fig. 5d), and the intraspecies force-constant fluctuations vary drastically depending on the bond lengths (see Supplementary Fig. 1). We emphasize that the force constants in AgPd depend mainly on the bond lengths and are relatively insensitive to the direct impact of the chemical environment. This was demonstrated by evaluating the species-pair-dependent force-constant distribution for the unrelaxed structure with all atoms fixed at their ideal lattice sites, i.e., making all bond lengths identical. This resulted in narrow force-constant distributions in which the magnitude of the force constants depends only on the bond length, as seen in Supplementary Fig. 2. Moreover, even though the linewidth magnitudes predicted by the full SPU simulations are much smaller than those for either NiCo or NiFe, the fractional disagreement of the species-averaged SPU-A linewidth is similar to that for NiFe. As a more complex example we show in Fig. 6b SPU and SPU-A linewidth simulations for the ternary $3 d$ equiatomic alloy, $\mathrm{NiFeCo}$, for which ICPA is not yet formulated. The large discrepancy between the SPU and SPU-A linewidth simulations predicts that the present ICPA Hamiltonian will again break down for linewidth predictions of $\mathrm{NiFeCo}$, analogous to those observed for NiFe. The SPU and SPU-A phonon spectral functions of $\mathrm{AgPd}$ and $\mathrm{NiFeCo}$ are shown in Supplementary Fig. 3.

Analyzing further, the magnitude and the wavevector, q, dependence of the simulated and measured linewidths for $\mathrm{NiCo}$ and NiFe (see Fig. 3) provides direct quantitative insight into the force-constant disorder-induced phonon linewidth $\Delta$ averaged over a length scale $\mathrm{I}=2 \pi / q$. In particular, linewidths of $\Delta=1 \mathrm{meV}$ correspond to phonon lifetimes of $0.66 \mathrm{ps}$, and $q=0.25 \mathrm{rlu}$ corresponds to I $\sim 15 \AA$. Accordingly, at low $q=0.25$ rlu, the phonon broadening is expected to be small (such as that observed for LA phonons for NiCo in Fig. 3) since longerwavelength phonons are expected to be less sensitive to local force- constant disorder. Surprisingly, however, for NiFe at $q=[0$, $0,0.25]$ rlu — corresponding to I $\sim 15 \AA$-the broadening of the LA mode is substantial and more than five times that of NiCo. This result is consistent with the observations in Fig. 5 of the remarkably broader force-constant distributions in NiFe than in NiCo. Moreover, by comparing SPU linewidths for NiFe with SPU-A simulations in Fig. 3, the intraspecies force-constant disorder (included in SPU but not in SPU-A) plays the dominant role in the NiFe phonon broadening at low- $q$. At large $q$ (near the $X$ point), where force-constant disorder is resolved on the scale of the fcc cubic cell, the measured and simulated phonon broadening for both $\mathrm{NiCo}$ and NiFe tend to increase (see Fig. 3), albeit not necessarily monotonically.

The results of this work have far-reaching implications. The discovery that local chemical environments play a dominant role in determining the impact of force-constant fluctuations in disordered alloys provides both fundamental insight on the underlying science of disordered alloys and technological insight toward potential atomic-level engineering-by tuning the types 

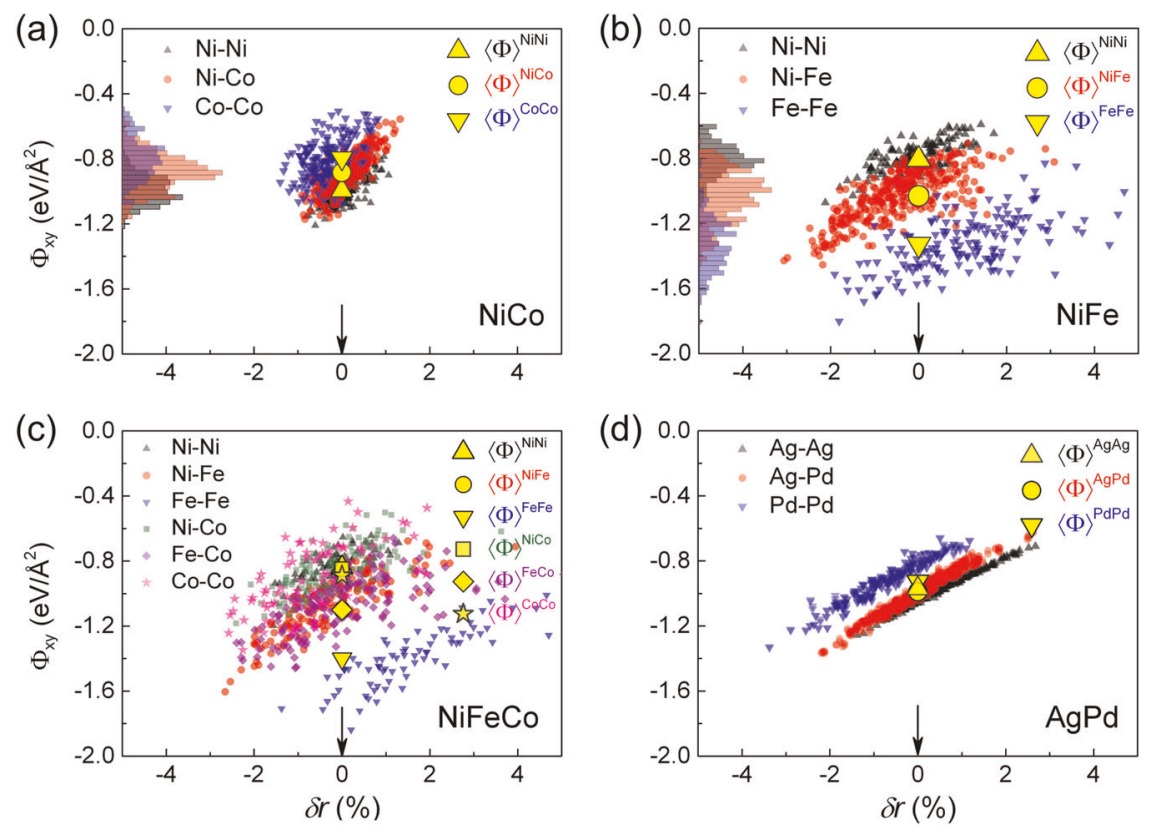

Fig. 5 Comparison of force-constant distributions. The $x y$ component of the nearest-neighbor force constant $\left(\Phi_{\mathrm{xy}}\right)$ versus percent variation of the bond lengths $(\delta \mathrm{r})$ in a NiCo, $\mathbf{b} \mathrm{NiFe}, \mathbf{c ~ N i F e C o}$, and $\mathbf{d} \mathrm{AgPd}$. The frequency counts of each component are shown in histograms on the $y$ axis. The yellow labels indicate the averaged values of the intraspecies force constants.

of alloying elements-to manipulate the microscopic vibrational physics in alloys. That is, the combination of theory and experimental measurements has demonstrated that the ab initio supercell approach combined with band unfolding provides an accurate description of the vibrational physics (phonon dispersion and lifetimes) of alloys with force-constant dominant disorder. Hence, SPU-calculated and experimentally measured linewidths can be used as predictors of phonon scattering and latticemediated macroscopic thermal transport in the design of novel materials such as that for new thermoelectric materials.

Finally, we emphasize that the physics of quasi-particles in disordered materials revealed in this study is applicable far beyond the scope of phonon excitations. For example, similar effects can be expected in magnetic alloys with broad, localenvironment induced distributions of exchange interactions. This was demonstrated in NiFeCoCr random solid solutions, ${ }^{38}$ where the broad distributions of species-pairwise Heisenberg exchange interactions calculated for this alloy could not be adequately represented by species-pair averages. In addition, the impact of random (off-diagonal) interatomic exchange (e.g., Heisenberg exchange, Dzyaloshinskii-Moriya interaction ${ }^{39,40}$ ) can be important for magnon dispersion and lifetimes, for low-energy control and magnon excitations in magnon spintronics, ${ }^{41}$ and for the helical period and dynamics of skyrmions in disordered helical magnets. ${ }^{42}$ Accordingly, the physics of force-constant disorder revealed here within the context of phonons is transferrable not only to random alloys with both mass and force-constant disorder, but also to the physics of other types of quasi-particles in disordered materials in which off-diagonal disorder is dominant, e.g., magnons or skyrmions in magnetic alloys. Thus, the potential for dominant, local-environment disorder effects, such as those reported for phonons in this study, provides a compelling case for further investigation across the rich variety of quasi-particle physics.

\section{METHODS}

\section{Experimental}

Phonon dispersion and linewidth measurements were performed on single-phase, single-crystal, solid-solution NiCo and NiFe samples (with negligibly small mass differences of $0.4 \%$ and $4.8 \%$, respectively) grown in an optical floating zone furnace. The measurements were performed at room temperature using high-resolution inelastic $X$-ray scattering (IXS) and inelastic neutron- scattering (INS) measurements along the [001] and [011] reciprocal lattice directions. For NiCo, IXS measurements were performed on the HERIX spectrometer at beamline 30-ID-C at the Advanced Photon Source at Argonne National Laboratory, and for NiFe INS measurements, were performed on the HB-3 triple-axis spectrometer at the High Flux Isotope Reactor at Oak Ridge National Laboratory (ORNL).

\section{Computational}

To explore the effect of force-constant disorder on the phonon properties of considered equiatomic alloys, we employed the projector-augmented wave method (PAW) ${ }^{43}$ implemented in the Vienna ab initio Simulation Package $(\mathrm{VASP})^{44}$ to evaluate the force constants using the standard supercell technique with the help of PHONOPY software. ${ }^{45}$ Spin-polarized calculations were performed in the spin colinear state except for AgPd. Exchange and correlation were treated using the generalized gradient approximation (GGA) parameterized by Perdew, Burke, and Ernzehof. ${ }^{46}$ The electron wave functions were expanded in a plane-wave basis set with cutoff kinetic energy set to $400 \mathrm{eV}$ for all calculations. Moreover, the disordered local environment was simulated explicitly using supercells constructed from special quasi-random structure $(\mathrm{SQS})^{47}$ without considering short-range order. A 「-centered $4 \times 4 \times 4(3 \times 3 \times 3)$ Monkhorst-Pack k-point mesh ${ }^{48}$ was used for Brillouin zone (BZ) integrations in multiple 64atom (108-atom) SQSs. The equilibrium atomic positions were obtained by optimizing the cell volume, and all internal degrees of freedom until the Hellmann-Feynman force on each atom are lower than 5 meV/Å. The cubic shape of the supercell is maintained while relaxing. The lattice parameters $\left(a_{0}\right)$ of $\mathrm{NiCo}, \mathrm{NiFe}, \mathrm{NiFeCo}$, and AgPd are 3.52, 3.57, 3.55, and $4.03 \AA$, respectively. This is consistent with previous experiments. ${ }^{49}$ Force constants of the supercells were calculated based on the optimized theoretical structures. The influence of spin noncolinearity on the force constants of ferromagnetic NiFe, NiFeCo, and NiCo alloys at finite temperature is neglected here. This is justified by the fact that all the ferromagnetic alloys considered here have high Curie temperatures as compared with room temperature, ${ }^{49}$ and therefore, the spin noncolinearity and its effect on the force constants are likely negligible. The phonon spectral functions of the random alloys were calculated using the itinerant coherent potential approximation (ICPA) and supercell phonon-unfolding (SPU) methods, as discussed separately later. For direct comparison with experiments, theoretical phonon spectral functions were convoluted with the $\boldsymbol{E}$ and $\boldsymbol{Q}$ resolution of the measurements. Then the same procedure 

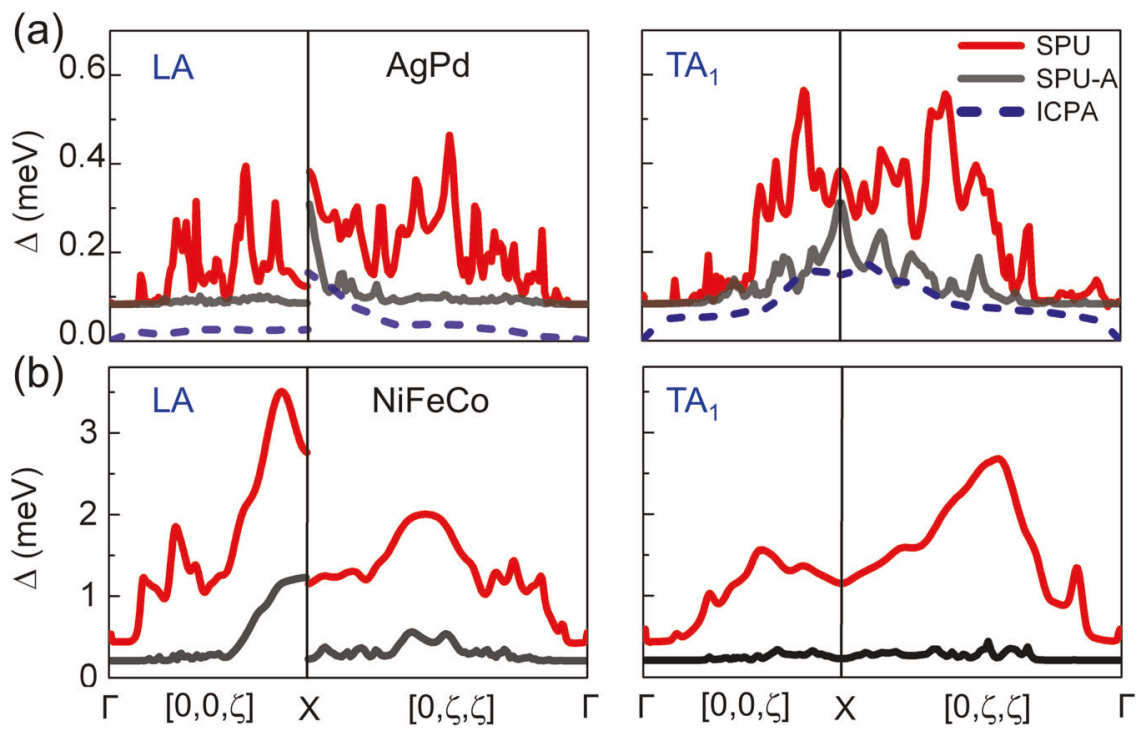

Fig. 6 Calculated phonon linewidths of other alloys. a AgPd and b NiFeCo.

that was used to extract linewidths from the experimental data was used to extract the theoretical linewidths from the convoluted theoretical phonon spectra.

The itinerant coherent potential approximation (ICPA). The itinerant coherent potential approximation (ICPA) ${ }^{19}$ is a Green's function-based formalism to calculate the phonon spectra in substitutionally disordered alloys. It extends the single-site coherent potential approximation (CPA) by considering scattering from more than one site embedded in an effective medium. Hence, all relevant disorders, i.e., the mass, the force constant, and the environmental disorders, are appropriately addressed within the ICPA. While calculating the configuration-averaged quantities, the method also ensures self-consistency, site-translational invariance, and analyticity of the Green's function. The phonon frequencies and the disorder-induced linewidths were determined, respectively, from the peaks and from the widths of the coherent scattering structure factor defined as

$$
S^{c}(\vec{q}, \omega)=\sum_{s, s^{\prime}} c_{s} c_{s^{\prime}} \frac{1}{\pi} \operatorname{Im} \ll G_{\lambda}^{s, s^{\prime}}\left(\vec{q}, \omega^{2}\right) \gg
$$

where $\lambda$ is the normal-mode branch index, $c_{s}$ is the coherent scattering length for species $s$, and $\frac{1}{\pi} \operatorname{Im} \ll G_{\lambda}^{s, s^{\prime}}\left(\vec{q}, \omega^{2}\right) \gg$ is the configuration-averaged and thermodynamics-averaged spectral function associated with the species pair $s, s^{\prime}$.

Supercell phonon-unfolding (SPU) method. In the supercell method for alloys, a particular finite-size supercell with defects breaks the space group symmetry and leads to a shrinking $B Z$ in reciprocal space. To recover the phonon spectra within the BZ of the primitive cell, state-of-the-art bandunfolding methods have been developed for electronic problems ${ }^{50,51}$ as well as for phonon problems. ${ }^{30-32}$ Here we use the unfolding program developed by Ikeda et al. to carry out the phonon band unfolding. According to the formula derived by lkeda et al., ${ }^{32}$ the phonon spectral function $A(\boldsymbol{k}, \omega)$ at the wavevector $\boldsymbol{k}$ and frequency $\omega$ is given as $A(\boldsymbol{k}, \omega)=\sum_{J} \| \hat{p}(k) v(K, J)^{2} \delta[\omega-\omega(K, J)]$. In this expression $\hat{p}(k)$ is the projection operator for wavevector $\boldsymbol{k}$ in the primitive $B Z, v(K, J)$ is the eigenvector of the dynamical matrix of the supercell for phonon mode $J$ at the $K$ point (defined in the reduced $\mathrm{BZ}$ ), and $\omega(K, J)$ is the corresponding eigenvalue. See ref. ${ }^{32}$ for further details.

Finally, the SPU-A calculations were performed for a single 256-atom supercell to capture aspects of the configurational averaging inherent to ICPA, whereas each of the full SPU phonon spectral function calculations presented (except for AgPd) represent averages over several (i.e., six for 64 and three for 108 atoms) supercells. The SPU phonon spectral function for AgPd was obtained from a single 108-atom supercell.

Connection between ICPA and SPU. The connection of the SPU with the ICPA can be established through the postulated ansatz that configurational averaging for an infinite random system can be approximated by manually averaging the observables (PDOS and spectral functions) over many finite SQS cells. A particular advantage of the SPU approach, however, is that it couples straightforwardly with density functional theory (DFT)-computed force constants without the need for any special averaging procedure. Hence, SPU has the required capability to provide complete information on the impact of force-constant disorder, i.e., the fluctuations between different atomic pairs as well as their full environment dependence. However, we note that so far, neither ICPA nor SPU has been tested for random alloys dominated by force-constant disorder.

\section{DATA AVAILABILITY}

The authors declare that the data supporting this study are available from the corresponding author upon request.

Received: 19 June 2019; Accepted: 19 December 2019; Published online: 17 January 2020

\section{REFERENCES}

1. Mühlbauer, S. et al. Skyrmion lattice in a chiral magnet. Science 323, 915-919 (2009).

2. Yu, X. Z. et al. Real-space observation of a two-dimensional skyrmion crystal. Nature 465, 901 (2010).

3. He, Q. L. et al. Chiral Majorana fermion modes in a quantum anomalous Hall insulator-superconductor structure. Science 357, 294-299 (2017).

4. Kasahara, Y. et al. Majorana quantization and half-integer thermal quantum Hall effect in a Kitaev spin liquid. Nature 559, 227 (2018).

5. Kasahara, Y. et al. Unusual thermal Hall effect in a Kitaev spin liquid candidate aRuCl 3. Phys. Rev. Lett. 120, 217205 (2018).

6. Kitaev, A. Anyons in an exactly solved model and beyond. Ann. Phys. 321, 2-111 (2006).

7. Ye, M., Halász, G. B., Savary, L. \& Balents, L. Quantization of the thermal Hall conductivity at small Hall angles. Phys. Rev. Lett. 121, 147201 (2018).

8. Soven, P. Coherent-potential model of substitutional disordered alloys. Phys. Rev. 156, 809-813 (1967).

9. Taylor, D. W. Vibrational properties of imperfect crystals with large defect concentrations. Phys. Rev. 156, 1017-1029 (1967).

10. Onodera, Y. \& Toyozawa, Y. Persistence and amalgamation types in the electronic structure of mixed crystals. J. Phys. Soc. Jpn. 24, 341-355 (1968).

11. Nickel, B. G. \& Butler, W. H. Problems in strong-scattering binary alloys. Phys. Rev. Lett. 30, 373-377 (1973).

12. Sanchez, J. M., Ducastelle, F. \& Gratias, D. Generalized cluster description of multicomponent systems. Phys. A Stat. Mech. its Appl 128, 334-350 (1984).

13. Gonis, A. \& Garland, J. W. Rederivation and proof of analyticity of the BlackmanEsterling-Berk approximation. Phys. Rev. B 16, 1495 (1977). 
14. Gonis, A. \& Garland, J. W. Self-consistent cluster theories for alloys with diagonal and off-diagonal disorder. Phys. Rev. B 18, 3999 (1978).

15. Lax, M. Multiple scattering of waves. Rev. Mod. Phys. 23, 287 (1951).

16. Stocks, G. M., Williams, R. W. \& Faulkner, J. S. Densities of states in Cu-Rich Ni-Cu alloys by the coherent-potential approximation: comparisons with rigid-band and virtual-crystal approximation. Phys. Rev. Lett. 26, 253-256 (1971).

17. Gyorffy, B. L. Coherent-potential approximation for a nonoverlapping-muffin-tinpotential model of random substitutional alloys. Phys. Rev. B 5, 2382 (1972).

18. Stocks, G. M., Temmerman, W. M. \& Gyorffy, B. L. Complete solution of the Korringa-Kohn-Rostoker coherent-potential-approximation equations: $\mathrm{Cu}-\mathrm{Ni}$ alloys. Phys. Rev. Lett. 41, 339 (1978).

19. Ghosh, S., Leath, P. L. \& Cohen, M. H. Phonons in random alloys: the itinerant coherent-potential approximation. Phys. Rev. B 66, 214206 (2002).

20. Mookerjee, A. A new formalism for the study of configuration-averaged properties of disordered systems. J. Phys. C. Solid State Phys. 6, L205 (1973).

21. Grånäs, O., Dutta, B., Ghosh, S. \& Sanyal, B. A new first principles approach to calculate phonon spectra of disordered alloys. J. Phys. Condens. Matter 24, 015402 (2011).

22. Dutta, B. \& Ghosh, S. Vibrational properties of $\mathrm{Ni}_{x} \mathrm{Pt}_{1-\mathrm{x}}$ alloys: an understanding from ab initio calculations. J. Appl. Phys. 109, 053714 (2011).

23. Dutta, B. \& Ghosh, S. The phonon spectra and elastic constants of $\mathrm{Pd}_{\mathrm{x}} \mathrm{Fe}_{1-\mathrm{x}}$ : an understanding from inter-atomic interactions. J. Phys. Condens. Matter 21, 095411 (2009).

24. Alam, A., Ghosh, S. \& Mookerjee, A. Phonons in disordered alloys: comparison between augmented-space-based approximations for configuration averaging to integration from first principles. Phys. Rev. B 75, 134202 (2007).

25. Dutta, B., Bisht, K. \& Ghosh, S. Ab initio calculation of phonon dispersions in sizemismatched disordered alloys. Phys. Rev. B 82, 134207 (2010).

26. Alam, A. \& Mookerjee, A. Vibrational properties of phonons in random binary alloys: an augmented space recursive technique in the $\mathrm{k}$ representation. Phys. Rev. B 69, 024205 (2004).

27. Alam, A., Chouhan, R. K. \& Mookerjee, A. Phonon modes and vibrational entropy of disordered alloys with short-range order: a first-principles calculation. Phys. Rev. B 83, 054201 (2011).

28. Blackman, J. A., Esterling, D. \& Berk, N. F. Generalized locator-coherent-potential approach to binary alloys. Phys. Rev. B 4, 2412 (1971).

29. Liechtenstein, A. II, Katsnelson, M. I., Antropov, V. P. \& Gubanov, V. A. Local spin density functional approach to the theory of exchange interactions in ferromagnetic metals and alloys. J. Magn. Magn. Mater. 67, 65-74 (1987).

30. Allen, P. B., Berlijn, T., Casavant, D. A. \& Soler, J. M. Recovering hidden bloch character: unfolding electrons, phonons, and slabs. Phys. Rev. B 87, 085322 (2013).

31. Boykin, T. B., Kharche, N., Klimeck, G. \& Korkusinski, M. Approximate bandstructures of semiconductor alloys from tight-binding supercell calculations. J. Phys. Condens. Matter 19, 36203 (2007).

32. Ikeda, Y., Carreras, A., Seko, A., Togo, A. \& Tanaka, I. Mode decomposition based on crystallographic symmetry in the band-unfolding method. Phys. Rev. B 95, 024305 (2017)

33. Popovici, M. On the resolution of slow-neutron spectrometers. IV. The triple-axis spectrometer resolution function, spatial effects included. Acta Crystallogr. Sect. A 31, 507-513 (1975).

34. Van De Walle, A. \& Ceder, G. The effect of lattice vibrations on substitutional alloy thermodynamics. Rev. Mod. Phys. 74, 11-45 (2002).

35. Ikeda, Y. et al. Temperature-dependent phonon spectra of magnetic random solid solutions. npj Comput. Mater. 4, 7 (2017).

36. Körmann, F., Ikeda, Y., Grabowski, B. \& Sluiter, M. H. F. Phonon broadening in high entropy alloys. npj Comput. Mater. 3, 36 (2017).

37. Ebert, $\mathrm{H}$. et al. Calculating linear-response functions for finite temperatures on the basis of the alloy analogy model. Phys. Rev. B 91, 165132 (2015).

38. Fukushima, T. et al. Local energies and energy fluctuations-applied to the high entropy alloy CrFeCoNi. J. Phys. Soc. Jpn. 86, 114704 (2017).

39. Dzyaloshinsky, I. A thermodynamic theory of "weak" ferromagnetism of antiferromagnetics. J. Phys. Chem. Solids 4, 241-255 (1958).

40. Moriya, T. Anisotropic superexchange interaction and weak ferromagnetism. Phys. Rev. 120, 91-98 (1960)

41. Chumak, A. V., Vasyuchka, V. I., Serga, A. A. \& Hillebrands, B. Magnon spintronics. Nat. Phys. 11, 453 (2015).

42. Nagaosa, N. \& Tokura, Y. Topological properties and dynamics of magnetic skyrmions. Nat. Nanotechnol. 8, 899 (2013).

43. Blöchl, P. E. Projector augmented-wave method. Phys. Rev. B 50, 17953-17979 (1994).

44. Kresse, G. \& Furthmüller, J. Efficient iterative schemes for ab initio total-energy calculations using a plane-wave basis set. Phys. Rev. B 54, 11169 (1996).

45. Togo, A. \& Tanaka, I. First principles phonon calculations in materials science. Scr. Mater. 108, 1-5 (2015).
46. Perdew, J. P. J., Burke, K. \& Ernzerhof, M. Generalized gradient approximation made simple. Phys. Rev. Lett. 77, 3865-3868 (1996).

47. Zunger, A., Wei, S.-H. S.-H., Ferreira, L. G. \& Bernard, J. E. Special quasirandom structures. Phys. Rev. Lett. 65, 353-356 (1990).

48. Monkhorst, H. J. \& Pack, J. D. Special points for Brillouin-zone integrations. Phys. Rev. B 13, 5188 (1976).

49. Jin, K. et al. Thermophysical properties of Ni-containing single-phase concentrated solid solution alloys. Mater. Des. 117, 185-192 (2017).

50. Popescu, V. \& Zunger, A. Effective band structure of random alloys. Phys. Rev. Lett. 104, 236403 (2010).

51. Ku, W., Berlijn, T. \& Lee, C.-C. Unfolding first-principles band structures. Phys. Rev. Lett. 104, 216401 (2010).

\section{ACKNOWLEDGEMENTS}

This work was supported as part of the Energy Dissipation and Defect Evolution (EDDE), an Energy Frontier Research Center funded by the U.S. Department of Energy (DOE), Office of Science, and Basic Energy Sciences under contract number DE-AC0500OR22725. This research used resources of the Advanced Photon Source, a DOE Office of Science User Facility operated by Argonne National Laboratory under Contract No. DE-AC02-06CH1 1357. Beamline support by Ayman Said of the Advanced Photon Source and Songxue Chi of the High Flux Isotope Reactor is acknowledged. This research also used resources at the High Flux Isotope Reactor, a DOE Office of Science User Facility operated by the Oak Ridge National Laboratory. This research used resources of Oak Ridge National Laboratory's Compute and Data Environment for Sciences (CADES) and the Oak Ridge Leadership Computing Facility, which is a DOE office of Science User Facility supported under Contract DE-AC05-00OR22725. Work at MPI was supported by Deutsche Forschungsgemeinschaft (Germany) within the priority programme SPP 1599. L.L. and T.B. acknowledge support from the U.S. Department of Energy, Office of Science, Basic Energy Sciences, and Materials Sciences and Engineering Division. B.D. and S.M. acknowledge Dr. Fritz Körmann and Dr. Yuji lkeda for fruitful discussions. S.M. is grateful to Dr. Xin Huang and Dr. Lisha Fan for graphic support.

\section{AUTHOR CONTRIBUTIONS}

S.M., R.O. and B.D. contributed equally to this work. R.O. performed the experimental measurements; H.B. and K.J. grew the single crystals and characterized the samples; S.M. carried out the first-principles calculations and phonon-unfolding simulations; B.D. performed ICPA simulations; S.M., B.C.L. and G.M.S. wrote the initial draft and all authors participated in discussions and contributed materially in finalizing the paper.

\section{COMPETING INTERESTS}

The authors declare no competing interests.

\section{ADDITIONAL INFORMATION}

Supplementary information is available for this paper at https://doi.org/10.1038/ s41524-020-0271-3.

Correspondence and requests for materials should be addressed to S.M. or G.M.S.

Reprints and permission information is available at http://www.nature.com/ reprints

Publisher's note Springer Nature remains neutral with regard to jurisdictional claims in published maps and institutional affiliations.

Open Access This article is licensed under a Creative Commons Attribution 4.0 International License, which permits use, sharing, adaptation, distribution and reproduction in any medium or format, as long as you give appropriate credit to the original author(s) and the source, provide a link to the Creative Commons license, and indicate if changes were made. The images or other third party material in this article are included in the article's Creative Commons license, unless indicated otherwise in a credit line to the material. If material is not included in the article's Creative Commons license and your intended use is not permitted by statutory regulation or exceeds the permitted use, you will need to obtain permission directly from the copyright holder. To view a copy of this license, visit http://creativecommons. org/licenses/by/4.0/.

(c) The Author(s) 2020 